\title{
Agronegocios
}

\section{Encadenamientos productivos y multiplicadores de empleo para la actividad económica cría de ganado vacuno a partir de la matriz insumo producto Costa Rica}

2012

Productive linkages and employment multipliers for the cattle

raising economic activity based on the input-output table of Costa

Rica 2012.

Sylvia Arguedas Villalobos ${ }^{1}$

Fecha de recepción: 08 de setiembre, 2018 Fecha de aprobación: 04 de diciembre, 2018

Arguedas Villalobos, S. (2019). Encadenamientos productivos y multiplicadores de empleo para la actividad económica cría de ganado vacuno a partir de la matriz de insumo productivo Costa Rica 2012. Revista E-Agronegocios, 5(1). https://doi.org/10.18845/rea.v5i1.4031

Vol. 5. №1. Enero-junio, 2019 DOI: https://doi.org/10.18845/rea.v5i1.4031 


\section{Resumen}

El presente artículo busca analizar la relevancia de la actividad económica cría de ganado vacuno, dentro de la economía costarricense. Para tal efecto, se aplican las metodologías basadas en el modelo insumo producto planteado por Chenery y Watanabe, (1958), Rasmussen (1963) y Schuschny (2005), para el cálculo de los encadenamientos directos, la identificación de los sectores clave para las economías y la generación de empleo directo y total respectivamente. Los resultados de estas aplicaciones, confirman que la actividad económica cría de ganado vacuno es una actividad clave para la economía costarricense. Además, a nivel de la industria agropecuaria, es la principal actividad generadora de empleos indirectos y totales, mientras que a nivel nacional se encuentra entre las primeras diez actividades económicas con mayor generación de empleo indirecto y total.

Palabras clave: Matriz insumo producto, encadenamientos productivos, empleo directo, empleo indirecto, cría de ganado vacuno.

\section{Abstract}

This paper aims to analyze the relevance of cattle raising economic activity in the Costa Rican economy. For this purpose, methodologies based on the input-output model proposed by Chenery, and Watanabe (1958), Rasmussen (1963) and Schuschny (2005) have been applied, calculating direct linkages, identifying economic key activities and estimating the generation of direct and total employment. The results of this process confirm that the cattle raising economic activity is a key activity for the Costa Rican economy. Furthermore, in agricultural industry, it is the main activity in terms of generation of indirect and total employment. Nationwide, it is among the top ten economic activities with greater indirect and total employment generation.

Key words: Input-output table, productive linkages, direct employment, indirect employment, cattle raising. 


\section{Introducción}

Para la década de 1960, según el Banco Central de Costa Rica (BCCR), la industria agropecuaria contribuyó al Producto Interno Bruto (PIB) del país con un $23 \%$ de su valor, por encima del comercio (21\%), la industria manufacturera (17\%) y los servicios (10\%). De acuerdo con Villasuso (2000), la industria agropecuaria para la década de 1950 tuvo un papel importante como generadora de empleos, ya que un $55 \%$ de la población económicamente activa era absorbida por esta industria.

Actualmente, de acuerdo con datos del BCCR, la industria agropecuaria representa aproximadamente un $6 \%$ del PIB, lo que demuestra una disminución en la participación relativa dentro de este indicador. Sin embargo, esto no implica que no aporte al desarrollo económico del país. Al contrario, se ha convertido en la principal industria proveedora de materia prima para otras actividades, lo que implica economías más encadenadas

Una de las actividades que mayor participación ha tenido dentro de la economía agrícola del país es la actividad ganadera. De acuerdo con el BCCR, para el año 2015, la actividad denominada cría de ganado vacuno $^{2}$ representó un $18,5 \%$ del valor agregado (VA) agropecuario total, siendo la de mayor peso, seguido por el cultivo de banano y la cría de pollos, los cuales representa un $13,2 \%$ y $8,3 \%$ del VA respectivamente.

Según lo anterior y haciendo uso de la Matriz Insumo Producto (MIP) 2012 para Costa Rica, como una herramienta de análisis económico cuantitativo, se plantea utilizar la metodología descrita por Chenery y Watanabe (1958), mencionados por Schuschny (2005), para conocer: ¿cuáles son las relaciones intersectoriales que genera la actividad cría de ganado vacuno con el resto de las industrias de la economía nacional?, y ¿cuál es el impacto que tiene sobre la generación de empleo y sobre los ingresos?. Lo anterior afirmando que, la actividad cría de ganado vacuno es una actividad clave para la economía costarricense, ya que genera importantes encadenamientos productivos con otras actividades económicas por medio de su consumo intermedio y su producción, y que, la actividad cría de ganado vacuno es una actividad con un gran potencial en la generación de empleo directo e indirecto por poseer una importante cantidad de vínculos con el resto de las actividades de la economía nacional.

El artículo se estructura en cinco secciones, la sección II presenta el referente teórico que sirve de base para la elaboración del estudio, en la sección III se detalla la metodología utilizada para la estimación de los encadenamientos productivos, así como la generación de empleo, la sección IV muestra los principales resultados y por último la sección $\mathrm{V}$ presenta las principales conclusiones y recomendaciones que se derivan del estudio realizado.

\section{Referente teórico}

Trabajos publicados por investigaciones nacionales y principalmente internacionales constituyen un marco de referencia para analizar, a través de la MIP 2012 por actividad económica para Costa Rica, las estructuras productivas más relevantes desde la perspectiva de los encadenamientos productivos y la generación de empleo directo e indirecto.

${ }^{2}$ En el Sistema de Cuentas Nacionales de Costa Rica, la actividad económica Cría de Ganado Vacuno (AE022), comprende los productos: cría y reproducción de ganado bovino (NP028) y producción de leche cruda de vaca (NP033). AE022 corresponde al código de la actividad económica, mientras que NP028 y NP033 corresponden a la nomenclatura de producto para Costa Rica (NPCR). 
Es importante mencionar que la MIP fue creada por Wassily Leontief en 1936. Esta matriz se obtiene a partir de las cuentas nacionales de un país. Es una tabla que muestra las relaciones de equilibrio entre la oferta (producción) y la demanda (consumo) de bienes y servicios de la economía (Hernández, G. 2012).

La MIP, permite caracterizar las propiedades estructurales de la economía tanto en producción como en consumo, analizar las interrelaciones entre los sectores productivos, medir los impactos directos e indirectos que genera cada actividad productiva y cuantificar el impacto que genera sobre toda la economía el aumento/disminución de la producción de un sector en particular, entre otras aplicaciones (De Miguel C., Pereira M. 2011).

La MIP está constituida por tres apartados:

- Transacciones intersectoriales o utilización intermedia: muestra los flujos de compras y ventas entre sectores y resume la actividad intermedia de la economía.

- El valor agregado: muestra los pagos sectoriales al capital y al trabajo para transformar los insumos en productos y los otros impuestos menos los subsidios a la producción.

- El destino de la producción o utilización final: muestra las transacciones para el uso sectorial de los productos elaborados, es decir, el consumo de los hogares, el consumo público, la inversión y la variación de existencias (Hernández, G. 2012).

\begin{tabular}{|c|c|c|c|c|c|c|c|c|c|c|c|}
\hline \multirow[b]{2}{*}{$\begin{array}{l}\text { Activi } \\
\text { dad } \\
\text { Econó } \\
\text { mica }\end{array}$} & \multicolumn{3}{|c|}{ Demanda Intermedia } & \multirow{2}{*}{$\begin{array}{l}\text { Total } \\
\text { Dema } \\
\text { nda } \\
\text { Inter } \\
\text { media }\end{array}$} & \multicolumn{5}{|c|}{ Demanda Final } & \multirow[b]{2}{*}{$\begin{array}{l}\text { Total } \\
\text { Dem } \\
\text { anda } \\
\text { Final }\end{array}$} & \multirow[b]{2}{*}{$\begin{array}{l}\text { Utiliz } \\
\text { ación } \\
\text { Total } \\
=\text { VBP }\end{array}$} \\
\hline & $\begin{array}{l}\text { Activi } \\
\text { dad } 1\end{array}$ & $\begin{array}{l}\text { Activi } \\
\text { dad i }\end{array}$ & $\begin{array}{l}\text { Activi } \\
\text { dad } n\end{array}$ & & $\begin{array}{l}\text { Cons } \\
\text { umo } \\
\text { Hoga } \\
\text { res }\end{array}$ & $\begin{array}{l}\text { Cons } \\
\text { umo } \\
\text { Gobi } \\
\text { erno }\end{array}$ & $\begin{array}{l}\text { Form } \\
\text { ación } \\
\text { bruta } \\
\text { de } \\
\text { capita } \\
\text { I fijo }\end{array}$ & $\begin{array}{l}\text { Variac } \\
\text { ión } \\
\text { existe } \\
\text { ncias }\end{array}$ & $\begin{array}{l}\text { Exporta } \\
\text { ciones }\end{array}$ & & \\
\hline $\begin{array}{l}\text { Activi } \\
\text { dad } 1\end{array}$ & $\mathrm{X} 11$ & $\mathrm{x} 1 \mathrm{j}$ & $\mathrm{x} 1 \mathrm{n}$ & - & Ch1 & Cg1 & 11 & $\mathrm{Z1}$ & E1 & Y1 & $\mathrm{X} 1$ \\
\hline $\begin{array}{l}\text { Activi } \\
\text { dad i }\end{array}$ & Xi1 & Xij & Xin & - & Chi & Cgi & li & $\mathrm{Zi}$ & $\mathrm{Ei}$ & Yi & $\mathrm{Xi}$ \\
\hline $\begin{array}{l}\text { Activi } \\
\text { dad n }\end{array}$ & $\mathrm{Xn1}$ & Xnj & Xnn & - & Chn & Cgn & $\ln$ & $\mathrm{Zn}$ & En & Yn & $\mathrm{Xn}$ \\
\hline Capita & EBE1 & EBEj & EBEn & & & & & & & & \\
\hline $\begin{array}{l}\text { Salari } \\
\text { os }\end{array}$ & REM1 & REMj & REMn & & & & & & & & \\
\hline $\begin{array}{l}\text { Impue } \\
\text { stos - } \\
\text { subsid } \\
\text { ios }\end{array}$ & T-SB1 & T-SBj & $\mathrm{T}-\mathrm{SBn}$ & & & & & & & & \\
\hline VBP & $\mathrm{X} 1$ & $\mathrm{Xj}$ & $\mathrm{Xn}_{\mathrm{n}}$ & & & & & & & & \\
\hline
\end{tabular}


Figura 1. Esquema matriz insumo producto. Adaptación de Hernández (2012).Xi: valor de la producción de la actividad económica $\mathrm{i}, \mathrm{Xij}$ : valor de la producción que la actividad económica i le vende a la actividad j como insumos para su producción., REMj: remuneración a los asalariados que paga la actividad j., EBEj: excedente de explotación de la actividad j., Tj: los impuestos pagados por la actividad j, Sbj: subsidios recibidos por la actividad j, Chi: valor de la producción de la actividad i vendida como bien de consumo a los hogares, Cgi: valor de la producción de la actividad i vendida al sector público. Zi: es el valor de la producción de la actividad i destinada a los inventarios. Ei: es el valor de la producción de la actividad i exportada al resto del mundo. li: es el valor de la producción de la actividad i vendida como bien de inversión a los empresarios residentes (formación bruta de capital). Yi: corresponde a la demanda final, y está conformada por Chi + Cgi + li + Zi + Ei .

Como parte de investigaciones previas, Hernández, G. (2012), a través de la MIP 2007 para Colombia y la aplicación del análisis de los multiplicadores de producto, concluye que muchos de los sectores de la economía colombiana orientan su producción a la oferta de productos finales y no tienen gran importancia como consumo intermedio. Sin embargo, los sectores de químicos, electricidad y gas, maquinaria y equipo de transporte son muy importantes porque su producción, además de ser un bien final, está orientada a la producción de los demás sectores.

Mientras tanto Pino (2004) utilizando la MIP 1996 para Chile realiza un análisis comparativo de las estructuras tecnológicas de la región del Bío-Bío y el país. Los principales hallazgos sugieren que las actividades que lideran la economía en la región debido a su capacidad de impacto y con un indicador superior al 1,7 son la pesca, la manufactura, electricidad, agua y gas; comercio, hoteles y restaurantes. La industria manufacturera presenta un mayor multiplicador de una expansión uniforme de la demanda, lo que indica que el resto de los sectores pueden crecer o decrecer debido a las situaciones que enfrente la actividad manufacturera.

Con respecto al índice de poder de dispersión, Pino (2004), indicó que todos los sectores económicos de la región, entre los cuales se puede citar el sector agropecuario, pesca extractiva, minería, industria manufacturera entre otros, tienen multiplicadores mayores a los del país, lo que significa que la capacidad de arrastre de los sectores en la región es superior para los sectores de la economía en su conjunto. Mientras que desde el punto de vista del índice de sensibilidad, sectores como la manufactura y la electricidad, transporte y comunicaciones y la pesca tienen una capacidad de arrastre mayor que la del país en conjunto. (Pino 2004, p.80).

Por su parte Cuenca (2008), en su artículo titulado El Sector de Ganadería Bovina en Colombia. Aplicación de Modelos de Series de Tiempo. Expone que la ganadería y el sector agrícola mantienen bajos enlaces hacia atrás y hacia delante, mientras que el sector manufacturero presenta fuertes enlaces hacia atrás y hacia adelante, de ahí radica la importancia de los sectores modernos en el desarrollo económico.

El Banco Central del Ecuador (2015), a través del análisis de la MIP para el año 2012, realiza una clasificación de los sectores de la economía ecuatoriana según las actividades económicas que los conforman, e identifica como sectores clave los dedicados a la producción de café cereza, sin tostar, no descafeinado y plantas de café; cacao elaborado sin edulcorantes; servicios de transmisión y distribución eléctrica; energía eléctrica; ganado bovino y leche fresca sin elaborar y cuero sin curtir y piel, entre otros. Esto significa que en el caso de un aumento de la demanda final de sus productos, conduciría a un incremento relativamente grande de la demanda final de los demás sectores.

En el ámbito costarricense, Chaverri (2016), a partir de la MIP Costa Rica 2011, identifica las industrias con mayores encadenamientos hacia atrás son los productos cárnicos y productos lácteos con un multiplicador del producto de 2,13 , esto sugiere que ante un cambio de una unidad en la demanda de productos cárnicos y productos lácteos, el efecto total sobre la producción será de 2,13 unidades, de las cuales 1,07 obedecen 
a efectos directos y 1,06 a efectos indirectos. Mientras que los otros servicios, comercio al por mayor y al detalle, finanzas y seguros y el transporte, tienen mayores encadenamientos hacia adelante.

Por su parte, Sánchez (2015), haciendo uso de MIP Costa Rica para el año 2011, expresa que, las actividades económicas que generan mayores niveles de empleo total son las de otros servicios (406 mil), comercio (404 mil) y cultivos alimentarios (193 mil) y que las actividades de otros servicios, comercio, cultivos alimentarios, construcción de edificios y otros productos alimenticios presentan mayores coeficientes de elasticidad empleo producto, lo que significa que un incremento del uno por ciento en la demanda final de la actividad denominada otros servicios, genera un incremento del 0.220 por ciento en el empleo total de la economía.

\section{Metodología}

El presente estudio utiliza información de la MIP por actividad económica del año 2012 elaborada por el Banco Central de Costa Rica. Se basa en investigaciones pioneras de Chenery y Watanabe (1958) y Hirschman y Rasmussen (1963), citados por Schuschny (2005) y Hernández (2012), los cuales proponen implementar el modelo insumo producto para establecer los encadenamientos productivos y a través de ellos, identificar los sectores clave de una economía

\section{El modelo de insumo producto}

A partir del modelo insumo-producto, se elabora un modelo simplificado de la economía, cuyas relaciones se establecen suponiendo una tecnología constante tanto en la producción de cada sector como en el consumo de cada bien o servicio. Se expresa en forma matricial de la siguiente manera:

$$
X=A * X_{i}+Y
$$

Donde $X$ es un vector tamaño $n x 1, n$ es el número de sectores de la economía, y $X_{i}$ es la producción del sector $i$. Seguidamente, $Y$ es un vector $n x m$, donde cada columna es cada uno de los componentes de la demanda final. Por último $A$ es una matriz $n x n$, denominada como matriz de requerimientos directos, ya que indica la proporción en que un insumo es demandado para generar una unidad de productos.

Los componentes $a_{i j}$ de la matriz $A$, son los coeficientes técnicos de la economía, estos permiten analizar la estructura sectorial. Se definen como:

$$
a_{i j}=\frac{X_{i j}}{X_{j}}
$$

Donde Xij es la producción de cada producto y Xj la producción total de los j productores.

Al sistema de ecuaciones descrito en (1), se le conoce como matriz de Leontief y se resuelve linealmente de la forma (3), donde la matriz $B=(I-A)^{-1}$ es la matriz de requerimientos totales de la economía (matriz de Leontief), y relaciona la producción de cada sector $X_{i}$ con la demanda final neta de importaciones.

$$
X=(I-A)^{-1} Y=B * Y
$$

Cada elemento de bij de la matriz de Leontief, representa la cantidad de producción que debería realizar el sector i-ésimo para satisfacer una unidad de demanda final neta de importaciones del producto j-ésimo $y$, como es constante da cuenta de la variación en el valor de la producción del sector i-ésimo como consecuencia de la demanda final neta de las importaciones del sector j-ésimo. 
En consecuencia los elementos de la matriz inversa cuantifican el impacto sobre la industria i-ésima de un cambio en la demanda final neta de importaciones del sector j-ésimo. Estos coeficientes capturan en un solo número efectos multiplicativos directos e indirectos, ya que cada sector afectado deberá impactar no sólo sobre sí, sino también sobre los demás que lo utilizan como insumo.

\section{Multiplicadores y encadenamientos ${ }^{3}$}

De acuerdo con Schuschny (2005), antes de hacer los análisis de encadenamientos se busca determinar la importancia relativa de los coeficientes, es decir, como un sector produce cambios importantes en su producción y en su demanda. Para esto se sigue el método desarrollado por Schintke y Staglin (1985), Sebal (1974) y Aroche (1996), citados por Schuschny (2005), donde un coeficiente técnico $a_{i j}$ es importante si una variación menor del $1 \%$ provoca un cambio mayor que un nivel prefijado p\% -suele tomarse el $0,5 \%$ o el $1 \%$ - en la producción total de alguno de los sectores. La fórmula para obtener la sensibilidad de los coeficientes es:

$$
w_{i j}(p)=a_{i j}\left(b_{i j} p+b_{i j} \frac{x_{j}}{x_{1}}\right)
$$

Donde $a_{i j}$ es el coeficiente técnico, $b_{j i}$ y $b_{i i}$ son elementos de la matriz de Leontief $(1), X_{j}$ y $X_{i}$ son las producciones respectivas de los sectores considerados. Cuanto más alto sea el valor de $w_{i j}$ más importante será el coeficiente $a_{i j}$. También se puede definir como:

$$
c_{i j}=\frac{p}{w_{i j}(p)}
$$

Por tanto, los coeficientes $a_{i j}$ más importantes tienen un bajo $c_{i j}$. Si el valor de $p$ es del $1 \%$, la tasa de variación del coeficiente técnico está dada por:

$$
c_{i j}=\frac{0,01}{a_{i j}\left(0,01 b_{i j}+b_{i j} \frac{x_{j}}{x_{1}}\right.}
$$

Hernández (2012) y basándose en lo planteado por Iraizoz y Garate (1999), sugiere que:

Cuanto más importante el coeficiente técnico $a_{i j}$ menor es el valor de $c_{i j}$, al indicar la variación máxima que puede tener el coeficiente a partir de la cual se altera la producción del sector en más del $1 \%$. Después de obtener los valores de $c_{i j}$ se utiliza la siguiente clasificación:

- Coeficientes muy importantes: $c_{\mathrm{ij}}<0,1$

- Coeficientes bastante importantes: $0,1 \leq c_{i j}<0,5$

- Coeficientes poco importantes: $0,5 \leq c_{i j}<1,0$

- Coeficientes no importantes: $c_{i j} \geq 1,0$

Esta clasificación implica que, la presencia de muchos coeficientes importantes en la fila, correspondientes a un determinado producto, indica que es muy importante como oferente de bienes de consumo intermedio para el proceso productivo de otros sectores. Mientras tanto, cuando ello ocurre en la columna, indica que el sector provoca aumentos de producción importantes en otras industrias para poder satisfacer su demanda de productos intermedios, reflejando la importancia del método de producción

\footnotetext{
${ }^{3}$ Basado en Schuschny, A.R. (2005). Tópicos sobre el Modelo de Insumo-Producto: teoría y aplicaciones.
} 
empleado por este sector, para la demanda de productos de otros sectores. Los coeficientes técnicos utilizados, tanto aij como bij, se construyen de acuerdo a (2) (Hernández, G. 2012).

\section{Encadenamientos directos}

Según Schuschny (2005), Chenery y Watanabe (1958) calculan los encadenamientos, con el fin de cuantificar el impacto directo de una rama sobre el resto de la economía, seleccionando aquellas actividades cuyos efectos eran superiores a la media combinando dos criterios:

- Encadenamientos directos hacia atrás: miden la capacidad de un sector de arrastrar directamente a otros ligados a él, por su demanda de bienes de consumo intermedio y estimulando a su vez la actividad de tales sectores. Se puede calcular como la proporción de las compras intermedias de un sector, en relación a sus producción efectiva:

$$
D B L_{j}=\frac{\sum_{i=1}^{n} X_{i j}}{X_{j}} \equiv \sum_{i=1}^{n} a_{i j}
$$

- Encadenamientos directos hacia adelante: miden la capacidad de un sector de estimular a otros, en virtud de tener su capacidad de oferta. Este indicador se mide como la fracción de sus ventas para consumo intermedio, sobre sus ventas totales.

$$
D F L_{i}=\frac{\sum_{j=1}^{n} X_{i j}}{X_{i}} \equiv \sum_{j=1}^{n} d_{i j}
$$

Dependiendo de los valores de $D B L$ y $D F L$, Chenery y Watanabe (1958) clasifican a los sectores en cuatro grupos:

Cuadro 1. Tipología sectorial según los multiplicadores directos de Chenery y Watanabe

\begin{tabular}{c|l|l}
\hline & \multicolumn{1}{|c|}{$D B L_{j}<\frac{\sum_{j=0}^{n} D B L_{j}}{n}$} & \multicolumn{1}{c}{$D B L_{j} \geq \frac{\sum_{i=0}^{n} D B L_{j}}{n}$} \\
\hline$D F L_{i} \geq \frac{\sum_{i=0}^{n} D F L_{i}}{n}$ & $\begin{array}{l}\text { No manufacturera / Destino } \\
\text { intermedio }\end{array}$ & $\begin{array}{l}\text { Manufacturera / Destino } \\
\text { intermedio }\end{array}$ \\
\hline$D F L_{i}<\frac{\sum_{i=0}^{n} D F L_{i}}{n}$ & No manufacturera / Destino final & Manufacturera / Destino Final \\
\hline
\end{tabular}

Fuente: Schuschny (2005).

- No manufactureras /Destino Intermedio: son sectores que venden a otros, cantidades sustantivas de su producción, y por eso poseen altos encadenamientos hacia adelante y bajos hacia atrás; corresponden a sectores de producción primaria intermedia.

- Manufactureras/Destino intermedio: son sectores que compran cantidades sustantivas de insumos, y venden su producción a otros sectores. Por esa razón, poseen altos encadenamientos hacia atrás y adelante. Desde el punto de vista de la articulación interna de malla productiva, son sectores más interesantes, ya que son responsables de propagar cualquier aumento de la demanda final. 
- Manufactureras/Destino final: se trata de sectores que compran a otros, cantidades sustantivas de insumos, pero que la mayor parte de su producción se dirige a la demanda final. Poseen altos encadenamientos hacia atrás y bajos hacia adelante.

- No manufactureras/Destino final: no compran significativamente a los demás sectores, ni les venden insumos, por eso son considerados producción primaria. Su producción se dirige primordialmente a abastecer la demanda final. Son sectores de bajos encadenamientos directos tanto hacia atrás como adelante.

\section{Encadenamientos totales}

De acuerdo con Schuschny (2005), para entender la interdependencia total entre los sectores, construyen índices agregados de encadenamiento hacia atrás o adelante. Esto significa, promediar pesadamente los índices de encadenamiento considerando la importancia relativa de cada sector, en la demanda o en los insumos primarios respectivamente:

$$
\begin{aligned}
B L_{\text {total }} & =\sum_{j=1}^{n} \alpha_{j} B L_{j} \\
F L_{\text {total }} & =\sum_{i=1}^{n} \beta_{i} F L_{i}
\end{aligned}
$$

Donde $\alpha_{j}=\frac{Y_{j}}{\sum_{i=0}^{n} Y_{i}}$, es decir, la participación del sector $j$ en la demanda total final neta de las importaciones y $\beta_{i}=\frac{V A B_{i}}{\sum_{j=0}^{n} V A B_{j}}$, la participación del sector $i$ en los insumos primarios totales y VAB es igual al valor agregado bruto.

\section{Identificación de los sectores clave}

Según cita Hernández, G. 2012, además de identificar los encadenamientos entre las industrias, es importante conocer cómo los impactos de un sector dado, se distribuyen o dispersan a través de toda la economía. Esto se determina a través de las medidas de dispersión propuestas por Rasmussen (1963).

La primera medida corresponde al poder de la dispersión, es decir, el efecto promedio de un sector en los demás, medido por el efecto de un incremento unitario de la demanda final de ese sector sobre el promedio de los efectos en toda la economía. El cual se obtiene de la siguiente manera

$$
\Psi_{j}=\frac{n}{B L_{j}} \sqrt{\frac{1}{n-1} \sum_{i=1}^{n}\left(b_{i j}-\frac{B L_{J}}{n}\right)^{2}}
$$

Un alto valor de $\psi \mathrm{j}$ implica que el sector compra insumos de pocos sectores de la economía, y viceversa. Cuanto menor es su valor mayor es el impacto de la variación en la producción, pues se dispersa entre muchos sectores y la concentración se reduce. El indicador muestra cuánto pesa el sector j en el sistema productivo. 
Mientras tanto un indicador de sensibilidad de la dispersión de forma análoga al encadenamiento hacia adelante.

$$
\tau_{i}=\frac{F L_{i}}{\left(\frac{\sum_{i} F L_{i}}{n}\right)} \equiv \frac{n \sum_{j} b_{i j}}{\sum_{i} \sum_{j} b_{i j}}
$$

Para un $\tau_{i}>1$, el efecto es superior al promedio de la economía, mientras que si $\tau_{i}<1$, el efecto es inferior a ese promedio. El índice mide cuán sensible es un sector, a cambios generales de la demanda, permite saber cuál sector es más sensible a cambios debido a choques de producción, empleo e ingresos.

Un valor relativamente grande del poder de dispersión $\pi_{\mathrm{j}}$, indica que un sector pesa sobre el resto de sectores en un grado considerable. Un sector de este tipo dependerá, en gran medida del resto de los sectores. Un "sector clave" con un valor de $\pi_{\mathrm{j}}$ grande y $\psi_{j}$ pequeño conduciría, en el caso de un aumento de la demanda final de sus productos, a un incremento relativamente grande de la demanda final de los demás sectores. A estos sectores se les denomina: sectores clave Tipo A. También para identificar sectores clave, se considera discriminar aquellos sectores, cuyos valores de $\pi_{\mathrm{j}}$ y $\tau i$ son ambos mayores a 1 (sectores clave Tipo B).

Cuadro 2. Identificación de Sectores clave Tipo A

\begin{tabular}{|c|l|l|}
\hline & \multicolumn{1}{|c|}{$\pi_{j}<1$} & \multicolumn{1}{|c|}{$\pi_{j} \geq 1$} \\
\hline$\Psi_{j} \approx \Psi_{j}^{\text {mín }}$ & $\begin{array}{l}\text { Sectores de bajos arrastre } \\
\text { disperso }\end{array}$ & Sectores clave \\
\hline$\Psi_{j} \gg \Psi_{j}^{\text {mín }}$ & $\begin{array}{l}\text { Sectores de bajo arrastre } \\
\text { concentrado }\end{array}$ & $\begin{array}{l}\text { Sectores con arrastre } \\
\text { concentrado }\end{array}$ \\
\hline
\end{tabular}

Fuente: Schuschny (2005).

Cuadro 3. Identificación de Sectores clave Tipo B

\begin{tabular}{|c|l|l|}
\hline & \multicolumn{1}{|c|}{$\pi_{j}<1$} & \multicolumn{1}{c|}{$\pi_{j}<1$} \\
\hline$\tau_{j}<1$ & Sectores estratégicos & Sectores clave \\
\hline$\tau_{j}<1$ & Sectores independientes & Sectores impulsores \\
\hline
\end{tabular}

Fuente: Schuschny (2005).

Los sectores con altos encadenamientos hacia atrás y adelante, son considerados como sectores clave (Tipo B), pues al ser fuertes demandantes y oferentes, son sectores de paso obligado de los flujos intersectoriales. Los sectores denominados como estratégicos, poseen baja demanda de insumos, pero abastecen sustantivamente de insumos a otros sectores. La denominación de estratégicos, apunta al hecho de que son sectores que pueden constituir posibles cuellos de botella productivos, frente a shocks de demanda. Los sectores impulsores o de fuerte arrastre, con bajos encadenamientos hacia delante y altos hacia atrás; son sectores impulsores de la economía, pues suelen poseer consumo intermedio elevado y una oferta de productos que, mayoritariamente, abastece la demanda final. Por ello, pertenecen a la última fase del proceso productivo. Los sectores considerados como independientes, consumen una cantidad poco significativa de insumos intermedios y dedican la producción a satisfacer, principalmente, a la demanda final. Se trata de sectores aislados, que no provocan efectos de arrastre significativos en el sistema económico, ni reaccionan en forma relevante ante el efecto de arrastre, provocado por las variaciones de la demanda intermedia de otros sectores. 


\section{Efectos y multiplicadores de empleo}

Los efectos y multiplicadores del empleo, capturan el impacto de los cambios de la demanda final neta de importaciones, sobre el nivel de empleo por sectores. El vector de efectos empleo para los sectores se puede definir como:

$$
\vec{E}^{l a b}=\vec{\Lambda}^{\prime} \cdot B \text { es decir } E_{j}^{l a b}=\sum_{i=1}^{n} \lambda_{i} b_{i j} \operatorname{con} \lambda_{i}=\frac{n_{i}}{X_{i}}
$$

Donde $n_{i}$ es el nivel de empleo del sector $i$, es decir, su número de empleados. Esto significa que $\lambda i$ es empleo del sector por cada colón de su producto, y suele denominarse como el coeficiente de requerimientos directos de empleo. Mide el impacto sobre el nivel de empleo, originado por un cambio unitario en la demanda final neta de importaciones del producto del sector $j$.

Así, también se puede definir el vector de multiplicadores de empleo:

$$
\vec{M}^{l a b}=\vec{\Lambda}^{\prime} \cdot B \cdot \lambda^{-1}=M_{j}^{l a c}=\sum_{i=1}^{n} \frac{\lambda_{i}}{\lambda_{j}} b_{i j} \text { con } \lambda_{i}=\frac{n_{i}}{X_{i}} y \lambda \text { la matriz diagonal asociada }
$$

Este multiplicador da cuenta del incremento total del empleo, como resultado de un cambio de la demanda final neta de importaciones, que da lugar a la creación de una unidad adicional de empleo en el sector $j$-ésimo.

\section{Resultados y discusión}

Los resultados que a continuación se presentan, se basan en el trabajo de investigación titulado Encadenamientos productivos y multiplicadores de empleo e ingreso para la actividad económica cría de ganado vacuno a partir de la matriz insumo producto Costa Rica 2012, realizado por Arguedas (2017).

\section{Análisis de los coeficientes técnicos y su sensibilidad}

El análisis de los coeficientes técnicos para la actividad cría de ganado vacuno, indica que la actividad en cuestión establece vínculos sectoriales con 96 actividades económicas desde el punto de vista de la demanda, dentro de las cuales se menciona la cría de ganado vacuno, los servicios de apoyo a la agricultura, ganadería y actividades postcosecha y los servicios de comercio. Mientras que por el lado de la oferta establece vínculos con 39 actividades, entre las principales se encuentran la elaboración y conservación de carne y embutidos de aves, la elaboración y conservación de carne y embutidos de ganado vacuno y porcino y otros tipos de carne y la elaboración de productos lácteos.

Sin embargo, el análisis de sensibilidad de los coeficientes indica que, al aumentar o disminuir la producción de carne o leche cruda de vaca, solamente los servicios de apoyo a la agricultura y las actividades veterinarias tendrán un impacto importante en su producción con el fin de poder satisfacer las necesidades de insumos de la actividad ganadera. Mientras que un aumento o disminución en la producción de carne o leche cruda de vaca influirá en las estructuras de producción de las actividades dedicadas a la elaboración y conservación de carne y embutidos de aves / elaboración y conservación de carne y embutidos de ganado vacuno y porcino y otros tipos de carne y con la elaboración de productos lácteos. 


\section{Encadenamientos directos y totales hacia atrás $(\mathrm{BL})$}

Según los resultados que arroja el análisis de los encadenamientos directos hacia atrás, la actividad cría de ganado vacuno genera mayores encadenamientos que el resto del $65 \%$ de las 29 actividades económicas que conforman la industria agrícola (Cuadro 4), por tanto, es capaz de arrastrar directamente a otros sectores ligados sectores a ella, por su demanda de bienes de consumo intermedio, estimulando a su vez la actividad de tales sectores.

Mientras tanto desde el punto de vista de los encadenamientos totales y el efecto multiplicador, a partir de la información contenida en el cuadro (4), se puede ver que por cada millón de colones que aumente la demanda final de la actividad cría de ganado vacuno, los encadenamientos producen un incremento de 1,65 millones de colones en la demanda total de la economía, 0,42 millones de colones debido a un efecto directo, o sea un efecto sobre las actividades relacionadas directamente y que sirven de insumo en su proceso productivo, y 1,23 millones de colones debido a un efecto indirecto, es decir un efecto sobre otras actividades económicas debido a la relación en la malla intersectorial.

Cuadro 41. Principales actividades económicas agropecuarias con mayores encadenamientos totales hacia atrás. Costa Rica 2012

\begin{tabular}{llrrr}
\hline Posición & Actividad económica & Total & Directo & Indirecto \\
\hline 1 & Cría de pollos & 2,06 & 0,63 & 1,43 \\
2 & Acuicultura marítima y de agua dulce & 1,93 & 0,57 & 1,36 \\
3 & Cría de cerdos & 1,87 & 0,53 & 1,34 \\
4 & Cultivo de caña de azúcar & 1,85 & 0,54 & 1,31 \\
5 & Cultivo de maíz & 1,83 & 0,55 & 1,28 \\
6 & Cultivo de follajes & 1,78 & 0,52 & 1,26 \\
7 & Cultivo de frijol & 1,74 & 0,49 & 1,25 \\
8 & Cultivo de melón & 1,71 & 0,48 & 1,22 \\
9 & Cultivo de papa & 1,67 & 0,44 & 1,22 \\
10 & Cultivo de sandía & 1,65 & 0,44 & 1,21 \\
11 & Cría de ganado vacuno & 1,65 & 0,42 & 1,23 \\
12 & Cultivo de flores & 1,60 & 0,40 & 1,20 \\
13 & Cultivo de cebolla & 1,58 & 0,40 & 1,19 \\
14 & Actividades de apoyo a la agricultura, la ganadería y & 1,57 & 0,38 & 1,19 \\
15 & actividades postcosecha & 1,55 & 0,40 & 1,15 \\
\hline
\end{tabular}

Fuente: Elaboración propia con base en datos de la MIP Actividad Económica-Actividad Económica para Costa Rica 2012.

\section{Encadenamientos directos y totales hacia adelante (FL)}


Con respecto a los cálculos realizados para obtener el DFL, se observa que, a diferencia de los DBL, cría de ganado vacuno tiene mayores encadenamientos hacia adelante que hacia atrás, tal y como se muestra en el cuadro (5), lo que significa que, desde la perspectiva de la oferta, presenta mayores impactos directos hacia adelante que el resto de las actividades económicas agropecuarias, por tanto, tiene una gran capacidad para estimular a otros sectores en virtud de su capacidad de oferta.

A diferencia de los efectos de los encadenamientos totales hacia atrás, la actividad presenta mayores encadenamientos como oferente de insumos a otras industrias, ubicándose como la tercera actividad más importante del sector agropecuario, por debajo de las actividades de apoyo a la agricultura y el cultivo de café.

El efecto multiplicador de los encadenamientos totales, en este caso hacia adelante, indica que el incremento en un millón de colones, de la demanda de la actividad cría de ganado vacuno induce a un incremento de 1,88 millones de colones en la oferta de la economía, 0,71 millones de colones debido a un efecto directo (un incremento en la producción esta misma actividad) y 1,17 millones de colones por un efecto indirecto, porque sirve de insumo a otros sectores, principalmente a "Elaboración y conservación de carne y embutidos de aves / Elaboración y conservación de carne y embutidos de ganado vacuno y porcino y otros tipos de carne" y "Elaboración de productos lácteos"(Cuadro 5).

Cuadro 5. Principales actividades económicas agropecuarias con mayores encadenamientos totales hacia adelante. Costa Rica 2012

\begin{tabular}{|c|c|c|c|c|}
\hline Posición & Actividad económica & Total & Directo & Indirecto \\
\hline 1 & $\begin{array}{l}\text { Actividades de apoyo a la agricultura, la ganadería } \\
\text { y actividades postcosecha }\end{array}$ & 4,09 & 1,00 & 3,09 \\
\hline 2 & Cultivo de café & 2,02 & 1,05 & 0,97 \\
\hline 3 & Cría de ganado vacuno & 1,88 & 0,71 & 1,17 \\
\hline 4 & Cultivo de arroz & 1,68 & 1,16 & 0,53 \\
\hline 5 & Cultivo de caña de azúcar & 1,60 & 0,84 & 0,76 \\
\hline 6 & Cría de otros animales & 1,37 & 0,46 & 0,91 \\
\hline 7 & Cultivo de palma africana (aceitera) & 1,36 & 1,00 & 0,36 \\
\hline 8 & Cría de pollos & 1,33 & 0,68 & 0,65 \\
\hline 9 & Acuicultura marítima y de agua dulce & 1,25 & 0,87 & 0,38 \\
\hline 10 & Silvicultura y extracción de madera y caza & 1,22 & 0,33 & 0,90 \\
\hline 11 & Propagación de plantas & 1,22 & 0,23 & 0,99 \\
\hline 12 & Cultivo de papa & 1,18 & 0,52 & 0,65 \\
\hline 13 & Cría de cerdos & 1,17 & 0,95 & 0,23 \\
\hline 14 & $\begin{array}{l}\text { Cultivo de otras frutas, nueces y otros frutos } \\
\text { oleaginosas }\end{array}$ & 1,14 & 0,40 & 0,74 \\
\hline 15 & Cultivo de piña & 1,13 & 0,10 & 1,03 \\
\hline
\end{tabular}

Fuente: Elaboración propia con base en datos de la MIP Actividad Económica-Actividad Económica para Costa Rica 2012. 


\section{Clasificación sectorial según los multiplicadores directos}

De acuerdo con el resultado de los indicadores de encadenamientos directos hacia atrás y hacia adelante, la actividad económica cría de ganado vacuno, junto con otras siete actividades agropecuarias, se clasifica sectorialmente como una actividad Manufacturera-Destino Intermedio. Esto significa que es un sector que compra cantidades considerables de insumos y su producción es vendida a otros sectores, lo que da como resultado la generación de altos encadenamientos hacia atrás y hacia adelante, por tanto, es responsable de propagar cualquier aumento de la demanda final.

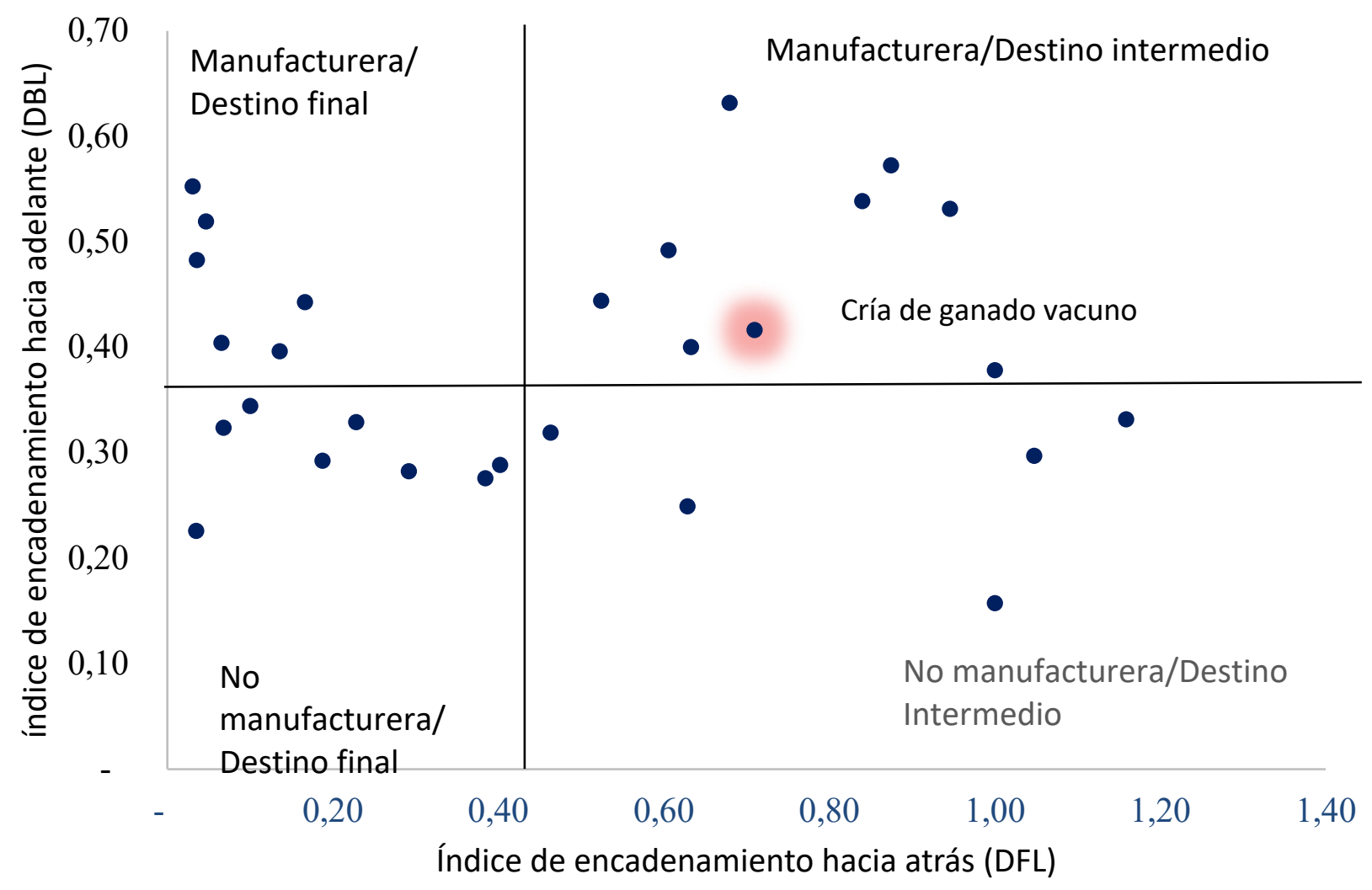

Figura 1. Tipología sectorial para la actividad económica cría de ganado vacuno, según Chenery y Watanabe. Costa Rica 2012.

Fuente: Elaboración propia con base en datos de la MIP Actividad Económica-Actividad Económica para Costa Rica 2012.

\section{Identificación de los sectores clave}

El poder de la dispersión y la sensibilidad de la dispersión son índices que permiten conocer cómo los impactos de un sector dado se distribuyen o dispersan a través de toda la economía y sirven para realizar una clasificación de los sectores de la economía, los que se clasifican como sectores clave, estratégicos, impulsores de la economía e islas (Cuadro 6).

La búsqueda de los "sectores clave" se basa en la suposición de que ciertas actividades económicas tienen el potencial de arrastrar o influenciar al resto, a través de los encadenamientos hacia atrás y hacia adelante que poseen con el resto de la economía.

Cuadro 6. Tipología sectorial según Rasmussen 


\begin{tabular}{l|ll}
\multicolumn{1}{c}{ PD $<1$} & PD $>1$ \\
\hline SP $>1$ & Estratégico & Clave \\
SP $<1$ & Isla & Impulsor
\end{tabular}

Fuente: Schuschny (2005).

Con respecto al índice del Poder de la dispersión (PD), la actividad económica cría de ganado vacuno, tal y como se muestra en el cuadro (7), posee un fuerte poder relativo de arrastre hacia atrás sobre el sistema productivo, ya que presenta un índice de poder la dispersión mayor a 1. En el cuadro también se aprecian las restantes 14 actividades económicas agropecuarias con alto poder de la dispersión.

Cuadro 2. Principales actividades económicas agropecuarias con mayores índices del poder de la dispersión. Costa Rica 2012

\begin{tabular}{lll}
\hline Posición & Actividad económica & PD \\
\hline 1 & Cría de pollos & 1,35 \\
2 & Acuicultura marítima y de agua dulce & 1,26 \\
3 & Cría de cerdos & 1,22 \\
4 & Cultivo de caña de azúcar & 1,21 \\
5 & Cultivo de maíz & 1,20 \\
6 & Cultivo de follajes & 1,16 \\
7 & Cultivo de frijol & 1,14 \\
8 & Cultivo de melón & 1,12 \\
9 & Cultivo de papa & 1,09 \\
10 & Cultivo de sandía & 1,08 \\
11 & Cría de ganado vacuno & 1,08 \\
12 & Cultivo de flores & 1,05 \\
13 & Cultivo de cebolla & 1,04 \\
14 & Actividades de apoyo a la agricultura, la ganadería y & 1,03 \\
& actividades postcosecha &
\end{tabular}

Fuente: Elaboración propia con base en datos de la MIP Actividad Económica-Actividad Económica para Costa Rica 2012.

Mientras tanto desde el punto de vista del índice de la Sensibilidad de la dispersión (SD), tal y como se puede ver en el cuadro (8), la actividad económica cría de ganado vacuno es una de las tres principales actividades económicas con índice de sensibilidad de la dispersión, lo implica que una expansión en el sistema de industrias afecta fuertemente a esta.

Cuadro 83. Principales actividades agropecuarias con mayores índices de la sensibilidad de la dispersión. Costa Rica 2012

\begin{tabular}{lll}
\hline Posición & Actividad económica & SD \\
\hline
\end{tabular}




\begin{tabular}{lll}
1 & $\begin{array}{l}\text { Actividades de apoyo a la agricultura, la ganadería y } \\
2\end{array}$ & 2,68 \\
3 & actividades postcosecha & 1,32 \\
4 & Cultivo de café & 1,23 \\
5 & Cría de ganado vacuno & 1,10 \\
6 & Cultivo de arroz & 1,04 \\
7 & Cultivo de caña de azúcar & 0,90 \\
8 & Cría de otros animales & 0,89 \\
9 & Cultivo de palma africana (aceitera) & 0,87 \\
10 & Cría de pollos & 0,82 \\
11 & Acuicultura marítima y de agua dulce & 0,80 \\
12 & Cropagación de plantas & 0,80 \\
13 & Cultivo de papa & 0,77 \\
14 & Cultivo de otras frutas, nueces y otros frutos oleaginosas & 0,74 \\
\hline
\end{tabular}

Fuente: Elaboración propia con base en datos de la MIP Actividad Económica-Actividad Económica para Costa Rica 2012.

Los datos anteriormente presentados muestran que la actividad cría de ganado vacuno posee índices de poder y sensibilidad de la dispersión mayores a 1, por tanto, se clasifica como una actividad clave para la economía del país. Esto significa que, al ser una industria con altos encadenamientos hacia atrás y hacia adelante, al darse un aumento de la demanda final de cualquier producto, sus requerimientos de insumo y producción aumentan en promedio más que el resto de los sectores, por lo que incentiva la producción de otras actividades relacionadas con ella. 


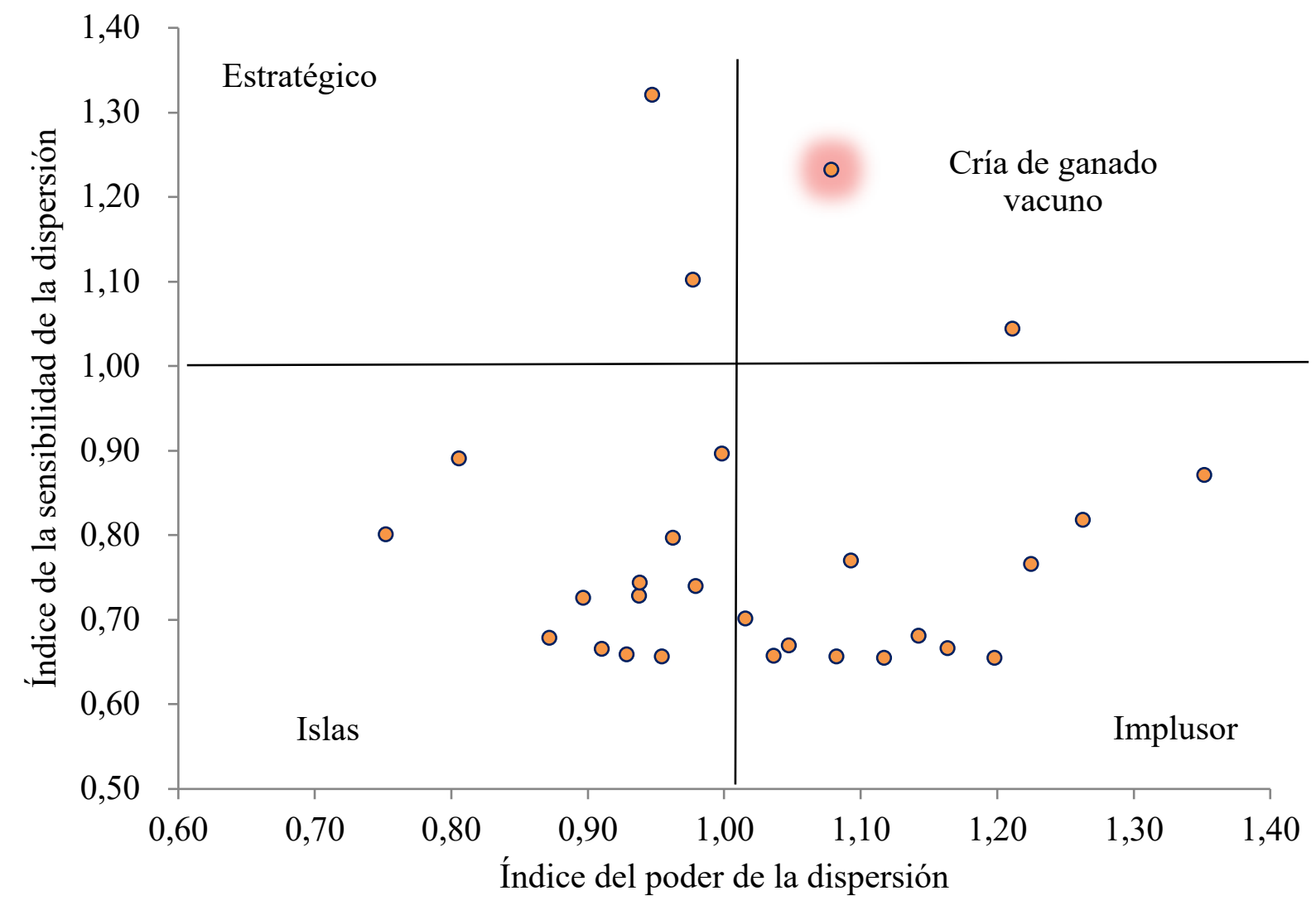

Figura 2. Tipología sectorial según Rasmussen para la actividad cría de ganado vacuno. Costa Rica 2012 Fuente: Elaboración propia con base en datos de la MIP Actividad Económica-Actividad Económica para Costa Rica 2012.

\section{Estimación de empleo directo y total}

Con respecto a la estimación de generación de empleo directo y total, dentro de la industria agropecuaria, la actividad cría de ganado vacuno es la actividad que mayor cantidad de puestos de trabajo indirectos y totales produce $(60,5$ mil puestos totales y 23,8 mil puestos indirectos), seguido de la actividad dedicada al cultivo de banano (60,4 mil puestos totales y 15 mil puestos indirectos), y el cultivo de café (52 mil puestos totales y 16 mil puestos indirectos) tal y como se observa en el cuadro (9).

Cuadro 9. Cantidad de puestos de trabajo totales, directos e indirectos para la industria agropecuaria. Costa Rica 2012

\begin{tabular}{|c|c|c|c|c|c|c|}
\hline \multirow{2}{*}{ Posición } & \multirow{2}{*}{ Actividad económica } & \multicolumn{5}{|l|}{ Empleo } \\
\hline & & Total & Directo & Indirecto & $\%$ directo & \%indirecto \\
\hline 1 & Cría de ganado vacuno & 60.584 & 36.781 & 23.803 & 0,61 & 0,39 \\
\hline 2 & Cultivo de banano & 60.462 & 45.396 & 15.066 & 0,75 & 0,25 \\
\hline 3 & Cultivo de café & 52.219 & 36.101 & 16.118 & 0,69 & 0,31 \\
\hline 4 & Cultivo de piña & 40.518 & 27.103 & 13.415 & 0,67 & 0,33 \\
\hline
\end{tabular}




\begin{tabular}{|c|c|c|c|c|c|c|}
\hline 5 & $\begin{array}{l}\text { Cultivo de otras hortalizas, } \\
\text { raíces o tubérculos n.c.p. }\end{array}$ & 30.952 & 21.616 & 9.336 & 0,70 & 0,30 \\
\hline 6 & $\begin{array}{l}\text { Actividades de apoyo a la agricultura, } \\
\text { la ganadería y actividades postcosecha }\end{array}$ & 15.955 & 10.155 & 5.800 & 0,64 & 0,36 \\
\hline 7 & Cultivo de palma africana (aceitera) & 14.781 & 12.013 & 2.768 & 0,81 & 0,19 \\
\hline 8 & $\begin{array}{l}\text { Cultivo de otras frutas, nueces y } \\
\text { otros frutos oleaginosas }\end{array}$ & 14.671 & 10.242 & 4.429 & 0,70 & 0,30 \\
\hline 9 & Cría de pollos & 13.500 & 6.538 & 6.962 & 0,48 & 0,52 \\
\hline 1( & Cultivo de frijol & 9.259 & 5.306 & 3.953 & 0,57 & 0,43 \\
\hline 1. & Resto de actividades agropecuarias & 77.475 & 49.692 & 27.783 & 0,64 & 0,36 \\
\hline
\end{tabular}

Fuente: Elaboración propia con base en datos de la MIP Actividad Económica-Actividad Económica para Costa Rica 2012.

A nivel de la economía total, la actividad en cuestión es la séptima industria generadora de empleos totales, al mismo tiempo es la quinta actividad con mayor cantidad empleo indirecto. Por otra parte, al producirse un aumento de un uno por ciento en la demanda final de la producción del país, cría de ganado vacuno aportará un incremento de 0,029 por ciento en el empleo total de la economía, lo que se traduce en de 606 nuevos puestos de trabajo de los cuales 368 son resultado de un efecto directo y 238 de un efecto indirecto. En el cuadro (10) se compara la posición que ocupa cría de ganado vacuno tanto contra otras actividades económicas con respecto a la generación de empleos totales y el aporte al mismo cuando hay cambios en la demanda total.

Cuadro 10. Efecto en el empleo total dado un aumento de un $1 \%$ en la demanda final de todas las actividades económicas. Costa Rica 2012

\begin{tabular}{|c|c|c|c|c|c|c|c|}
\hline \multirow{2}{*}{$\begin{array}{l}\text { Posic } \\
\text { ión }\end{array}$} & \multirow{2}{*}{ Actividad económica } & \multicolumn{3}{|c|}{ Empleo } & $\begin{array}{l}\Delta 1 \% \text { en la } \\
\text { demanda total }\end{array}$ & \multirow{2}{*}{$\begin{array}{l}\text { Emple } \\
\text { os } \\
\text { gener } \\
\text { ados }\end{array}$} & \multirow{2}{*}{$\begin{array}{l}\text { Elastic } \\
\text { dad }\end{array}$} \\
\hline & & Total & $\begin{array}{l}\text { Direc } \\
\text { to }\end{array}$ & $\begin{array}{l}\text { Indire } \\
\text { cto }\end{array}$ & $\begin{array}{l}\text { (mills. } \\
\text { colones) }\end{array}$ & & \\
\hline 1 & Comercio & $\begin{array}{l}575.2 \\
66\end{array}$ & $\begin{array}{l}385.5 \\
16\end{array}$ & $\begin{array}{l}189.7 \\
50\end{array}$ & $33.359,05$ & 5753 & 0,2765 \\
\hline 2 & Enseñanza & $\begin{array}{l}178.9 \\
96\end{array}$ & $\begin{array}{l}148.0 \\
39\end{array}$ & $\begin{array}{l}30.95 \\
7\end{array}$ & $21.240,00$ & 1790 & 0,086 \\
\hline 3 & Construcción de edificios & $\begin{array}{l}159.9 \\
28\end{array}$ & $\begin{array}{l}96.28 \\
4\end{array}$ & $\begin{array}{l}63.64 \\
4\end{array}$ & $20.512,42$ & 1599 & 0,0769 \\
\hline 4 & $\begin{array}{l}\text { Actividades de los hogares en } \\
\text { calidad de } \\
\text { empleadores de personal } \\
\text { doméstico }\end{array}$ & $\begin{array}{l}145.1 \\
28\end{array}$ & $\begin{array}{l}145.1 \\
28\end{array}$ & - & $2.823,38$ & 1451 & 0,0698 \\
\hline
\end{tabular}




\begin{tabular}{|c|c|c|c|c|c|c|c|}
\hline 5 & $\begin{array}{l}\text { Actividades de servicio de } \\
\text { comida y bebidas }\end{array}$ & $\begin{array}{l}130.3 \\
56\end{array}$ & $\begin{array}{l}74.30 \\
8\end{array}$ & $\begin{array}{l}56.04 \\
8\end{array}$ & $10.428,60$ & 1304 & 0,0627 \\
\hline 6 & $\begin{array}{l}\text { Actividades de atención de la } \\
\text { salud humana } \\
\text { y de asistencia social }\end{array}$ & $\begin{array}{l}106.1 \\
54\end{array}$ & $\begin{array}{l}83.72 \\
9\end{array}$ & $\begin{array}{l}22.42 \\
5\end{array}$ & $19.541,77$ & 1062 & 0,051 \\
\hline 7 & Cría de ganado vacuno & $\begin{array}{l}60.58 \\
4\end{array}$ & $\begin{array}{l}36.78 \\
1\end{array}$ & $\begin{array}{l}23.80 \\
3\end{array}$ & $3.625,61$ & 606 & 0,0291 \\
\hline 8 & Cultivo de banano & $\begin{array}{l}60.46 \\
2\end{array}$ & $\begin{array}{l}45.39 \\
6\end{array}$ & $\begin{array}{l}15.06 \\
6\end{array}$ & $4.151,75$ & 605 & 0,0291 \\
\hline 9 & $\begin{array}{l}\text { Actividades administrativas y de } \\
\text { apoyo de oficina }\end{array}$ & $\begin{array}{l}59.24 \\
6\end{array}$ & $\begin{array}{l}45.07 \\
4\end{array}$ & $\begin{array}{l}14.17 \\
2\end{array}$ & $7.406,61$ & 592 & 0,0285 \\
\hline 10 & $\begin{array}{l}\text { Mantenimiento y reparación de } \\
\text { vehículos }\end{array}$ & $\begin{array}{l}57.52 \\
4\end{array}$ & $\begin{array}{l}40.59 \\
9\end{array}$ & $\begin{array}{l}16.92 \\
5\end{array}$ & $3.745,35$ & 575 & 0,0277 \\
\hline
\end{tabular}

Fuente: Elaboración propia con base en datos de la MIP Actividad Económica-Actividad Económica para Costa Rica 2012.

\section{Conclusiones}

Desde el punto de vista de los encadenamientos productivos hacia atrás y hacia adelante, la actividad cría de ganado vacuno es una actividad que posee un fuerte poder para generar cambios sobre un grupo de industrias, estimulando la producción de estas a través de la demanda de insumos como consumo intermedio y como oferente de materia primaria.

A través del resultado de los encadenamientos directos hacia atrás y hacia adelante, la industria ganadera se clasifica como una actividad manufacturera de destino intermedio, la cual compra una cantidad considerable de insumos para su producción, que a su vez es vendida a otros sectores.

Los encadenamientos directos hacia adelante son más importantes que los encadenamientos directos hacia atrás, esto debido a que la actividad representa la principal fuente de insumos para la producción de las actividades dedicadas a la elaboración y conservación de la carne y embutidos de aves, elaboración y conservación de carne y embutidos de ganado vacuno y la elaboración de productos lácteos. Sin embargo, los encadenamientos indirectos hacia adelante son más fuertes, esto se debe a que la producción de las industrias mencionadas anteriormente también es insumo básico para la producción de otras industrias. Por tanto, en la medida en que se vea afectada la producción de leche o carne, el resto de las industrias verán un efecto indirecto en su estructura de costos de producción.

Las medidas de dispersión, representadas por el Poder de la dispersión y la Sensibilidad de la dispersión, tienen una gran relación con los encadenamientos productivos, ya que al igual que con los encadenamientos hacia atrás y hacia adelante, el índice del poder de la dispersión, es menor que el de la sensibilidad de la dispersión, lo que verifica que la actividad influye fuertemente en otras actividades desde 
el punto de vista de la oferta y no tanto desde la demanda. De cualquier forma, la actividad cría de ganado vacuno presenta indicadores por encima de la media del sistema productivo nacional, lo que confirma que es una actividad clave para la economía costarricense porque genera importantes encadenamientos productivos por medio de su demanda de insumos para consumo intermedio y por su oferta de producción utilizada como materia prima en otras actividades económicas.

En cuanto a la generación de empleo directo e indirecto, la actividad cría de ganado es una actividad estratégica, ya que dentro de la industria agropecuaria ocupa la primera posición, mientras que a nivel nacional es la sexta en cuanto a empleos totales. La actividad presenta mayor generación de empleo directo que indirecto, por lo tanto, se presume que, a diferencia del comportamiento de su producción, es una actividad menos encadenada.

Un aspecto importante por mencionar es que la metodología aplicada no había sido utilizada en ningún análisis de la industria agropecuaria. De la misma forma, en ninguno de los pocos estudios existentes que aplican esta metodología sobre otras ramas, se ha utilizado la MIP 2012, siendo esta la más reciente disponible de forma oficial. Lo anterior incrementa el aporte y la relevancia de las conclusiones obtenidas.

Según lo anterior, se considera de suma importancia que la información obtenida sirva como complemento al momento de preparar y evaluar futuros proyectos productivos, así como en el análisis de las cadenas valor de las diferentes industrias agropecuarias, especialmente para la actividad en cuestión.

Es recomendable que, el análisis llevado a cabo y sus conclusiones, sean valorados por los tomadores de decisiones de las instituciones que velan por un desempeño óptimo de la actividad ganadera del país, como una opción para el diseño de políticas no solo de generación de empleo, sino también como base para estrategias productivas y comerciales que fortalezcan al sector y resalten su importancia tal y como se demostró en este trabajo.

Es importante recalcar que la potencial aplicación de esta metodología para obtener resultados más actualizados está condicionada a la publicación de una nueva matriz por parte de fuentes oficiales, o bien a un esfuerzo de investigación futuro para construir una matriz actualizada a partir de los datos existentes.

\section{Literatura citada}

Arguedas, S.M. (2017). Encadenamientos productivos y multiplicadores de empleo e ingreso para la actividad económica cría de ganado vacuno a partir de la matriz insumo producto Costa Rica 2012. (Tesis de Licenciatura en Economía Agrícola con énfasis en Agroambiente) Universidad de Costa Rica, Sede Rodrigo Facio.

Banco Central de Costa Rica. (2016). Cuentas Nacionales período de referencia 2012. Presentación Cambio de Año Base, período de referencia 2012. Recuperado de http://www.bccr.fi.cr/ [Consulta 20 feb. 2016].

Banco Central del Ecuador. (2015). Matriz Insumo Producto. Herramienta fundamental de la contabilidad Nacional. Recuperado de http://www.bce.fin.ec/ [Consulta 28 feb. 2016].

Banco Mundial. (2005). Más allá de la ciudad: la contribución del campo al desarrollo. Recuperado de http://web.worldbank.org/ [Consulta 28 feb. 2016].

Chaverri, C. (2016). Encadenamientos productivos: una aplicación a partir de los datos de la Matriz Insumo Producto. Recuperado de http://estadonacion.or.cr/files/biblioteca_virtual/[Consulta 04 nov. 2016].

Chenery y Watanabe, (1958). An International Comparison of the Structure of Production. Econométrica, vol. 26, 4 de octubre 1958, pp. 487-521. 
Cuenca, N., Chavarro, F. y Díaz, O. (2008). El Sector de Ganadería Bovina en Colombia. Aplicación de Modelos de Series de Tiempo. Revista Facultad de Ciencias Económicas. Universidad Militar Nueva Granada. 16 (1). Recuperado de http://www.scielo.org.co/ [Consulta 01 nov. 2015].

De Miguel, C. y Pereira, M. (2011). Análisis de Insumo Producto. Comisión Económica para América Latina y el Caribe. Recuperado de http://www.cepal.org/ccas/noticias/paginas/1/45611/07_Matriz_de_Insumo_Producto_y_MEG.pdf. [Consulta 02 nov. 2015].

Hernández, G. (2012). Matrices Insumo-Producto y Análisis de Multiplicadores. Una Aplicación para Colombia. Revista de Economía Institucional. Universidad Externado de Colombia. 14 (26), 203-221. Recuperado de http://www.economiainstitucional.com/ [Consulta 01 nov. 2015].

Instituto Nacional de Estadística y Censos. (2015). VI Censo Nacional Agropecuario 2014. Resultados Generales. Recuperado de http://www.inec.go.cr/ [Consulta 02 nov. 2015].

Pino, O. (2004). Análisis de los Encadenamientos Productivos de la Economía. Base 1996. Revista Theoría. Universidad del Bío-Bío. 13, 71-82. Recuperado de http://www.ubiobio.cl/theoria/ [Consulta 01 nov. 2015].

Rasmussen, P. N. (1963). Relaciones intersectoriales, Editorial Aguilar, Madrid.

Sánchez, M.E. (2015). Identificación de sectores económicos con alto potencial en la generación de empleo a partir de la matriz insumo producto para Costa Rica 2011 . Recuperado de http://estadonacion.or.cr/files/biblioteca_virtual/ [Consulta 18 feb. 2016].

Schuschny, A.R. (2005). Tópicos sobre el Modelo de Insumo-Producto: teoría y aplicaciones. Chile: CEPAL Recuperado de http://www.cepal.org/ [Consulta 18 feb. 2016].

Villasuso, J.M. (2000). Reformas Estructurales y Política Económica en Costa Rica. Recuperado de http://repositorio.cepal.org/ [Consulta 28 feb. 2016] 